\title{
REFLEXÕES SOBRE A INFOGRAFIA: DAS NARRATIVAS ÀS SIMULAÇÕES
}


O presente texto apresenta reflexões desenvolvidas ao longo do processo de pesquisa em Estética da Interação no programa de pós-graduação da Universidade Federal de Pernambuco, tentando trazer à luz aquilo que, no campo da filosofia, tratamos conceitualmente como potência, e mais especificamente no que ela tange um dos tópicos em evidência do design de informação atualmente: a visualização de dados. Nesta abordagem, o que está realmente em discussão não é a superfície sensível e perceptível destes artefatos, e sim as questões lhes são estruturantes, dentro de se pode qualificar como um olhar tipicamente estruturalista: buscamos entender o que a infografia, em sua modalidade digital (também chamada de dataviz), poderia se tornar na construção da informação e em nossa relação com a produção de sentido e significação. 
Há uma ênfase muito recente sobre o papel dos dados em diversas práticas. 0 jornalismo de dados, ciências de dados, visualização de dados e, por que não, o design de dados, são colocados como novas formas de se usar a informação para nortear os campos de atuação. É também uma nova maneira de examinar as informações. Geralmente consideramos as informações como dados brutos após o processamento e, quando dizemos dados, geralmente queremos dizer muito deles. Neste universo de dados em quantidades massivas, subentende-se como informação algo que passou por um trabalho de ordenação ou organização intencional, que só assim permitiria às pessoas elaborarem um sentido sem ficarem desorientadas por sua demasia.

Para nos ajudar a dar sentido e compreender as coisas, os dados, que são tudo o que podemos perceber e documentar de alguma forma, são conformados em informação. O que Vilém Flusser chama de informação é esta atividade de dar materialidade (forma) as coisas que não as têm. Em seu ensaio "Forma e Material", trabalhando com uma visão platônica sobre modelos conceituais e coisas materiais, ele afirma:
Os projetos estariam sempre voltados para informar. E se a 'forma' for o 'como' da matéria e a 'matéria' for o 'o que' da forma, então o design é um dos métodos de dar forma a matéria e de fazê-la aparecer como aparece, e não de outro modo. 0 design, como todas as expressões culturais, mostra que a matéria não aparece (é inaparente), a não ser que seja informada (FLUSSER, 2007, p. 28).

O dataviz, corruptela de data visualization, é uma forma de informar, ou seja, de criar informação, organizando os dados processados de maneira visualmente compreensível. Isso não é novo. Mas há algo que pode ser novo nos métodos de modelagem de informações em um mundo onde a computação é ubíqua. Não só podemos apresentar dados brutos de forma compreensível e compacta, contornando sua interpretação por pessoas que, como prática comum, tendem a deformá-los para diversos fins, como também, o que é considerado 
uma grande quantidade de dados chega a um novo nível (denominado "Big Data"), lidando com uma escala de informação que os equipamentos de informática de 20 anos atrás não teriam poder computacional suficiente para sequer armazenar.

O objetivo desta análise não é criticar ou questionar esses novos campos gravitando a informação como mídia, mas discutir o potencial latente da visualização de dados, que muitas vezes é usada apenas como uma ornamentação para as informações, mas que, como tentaremos argumentar, está ontologicamente ligada à lógica da simulação, ainda que isto não seja um fato evidente para todos que trabalham com ela.

\section{A INFOGRAFIA ANTES DE UMA ABORDAGEM CENTRADA NA NARRATIVA}

Infografia ainda é um conceito em discussão no design de informação. Há autores que entendem todo tipo de informação representada de maneira pictórica como infografia, e há aqueles que consideram esta formulação vaga para o recorte de um tipo de artefato muito específico. Não é diferente na discussão entre os teóricos da computação, onde temos aqueles que entendem que qualquer informação apresentada na tela, ou impressa, é uma forma de visualizar dados; e outros que tratam como visualização de dados uma categoria delimitada da expressão computacional. 0 que trataremos como infografia aqui se limita a um conjunto pequeno de artefatos esquemáticos utilizados na produção editorial para comunicar, de forma geralmente lúdica e compacta, informações que necessitam ser correlacionadas para que possam constituir significação. Igualmente, não trataremos por dataviz interfaces gráficas ou outros tipos de representação digital oriundas de qualquer tipo de dado armazenado na memória de um computador, nos limitando àquelas cujo papel é voltado à construção de sentido (e não a sinalizar instruções, formas de uso, ou informações sobre o estado do próprio aparato computacional).

Os exemplos notáveis da infografia que instituem uma ideia generalizada do que ela é - aquilo que Wittgenstein (1987) chama de protótipo - são constituídos por ilustrações lindamente trabalhadas, representando informações de uma maneira lúdica e tentando contar uma história por meio do uso de metáforas e outras alegorias simbólicas. Essa já é uma maneira sofisticada de configurar o artefato de informação, aplicando conhecimentos sobre as relações subjacentes entre ludicidade, narrativa e retórica visual.

É quase seguro dizer que "Envision information" (1990), de Edward Tufte, é um marco significativo para isso. Tufte já era conhecido na área por "The visual display of quantitative information" (1983), um trabalho que, em suas próprias palavras, começou em "1975, quando Dean Donald Stokes, da Woodrow Wilson School de Princeton, me pediu para ensinar estatística a uma dúzia de jornalistas que estavam visitando aquele ano para aprender um pouco de 
economia."01 (TUFTE, 2001, p. 8). Seu traba-

Iho no primeiro livro ajudou os trabalhadores da mídia (primeiro) a entender e (depois) a criar representações visuais de dados: para trazer visualidade às notícias impressas, como meio de enriquecer a informação e fornecer uma retórica visual reforçando os argumentos verbais.

Os charts (imagens representando graficamente as informações numéricas) eram usados, na época, para facilitar o que os jornais consideravam uma operação cognitiva difícil de se solicitar aos seus leitores no processo de leitura (ou varredura) apressada das notícias: principalmente no que diz respeito à correlação de duas - ou às vezes mais - variáveis cuja construção de significados só seria possível através de suas interações. Essa correlação provavelmente poderia ser descrita por uma fórmula matemática simples, mas expressá-la graficamente diminuiria o esforço cognitivo necessário. Os charts são, como diz o título do livro de Tufte, uma representação simples de valores e cálculos e, portanto, elementos centrados em números.

01 “1975 when Dean Donald Stokes of Princeton's Woodrow Wilson School asked me to teach statistics to a dozen journalists who were visiting that year to learn some economics." (tradução do autor) 
Figura 1: Representação visual de informações numéricas em forma de charts. Fonte: do autor.

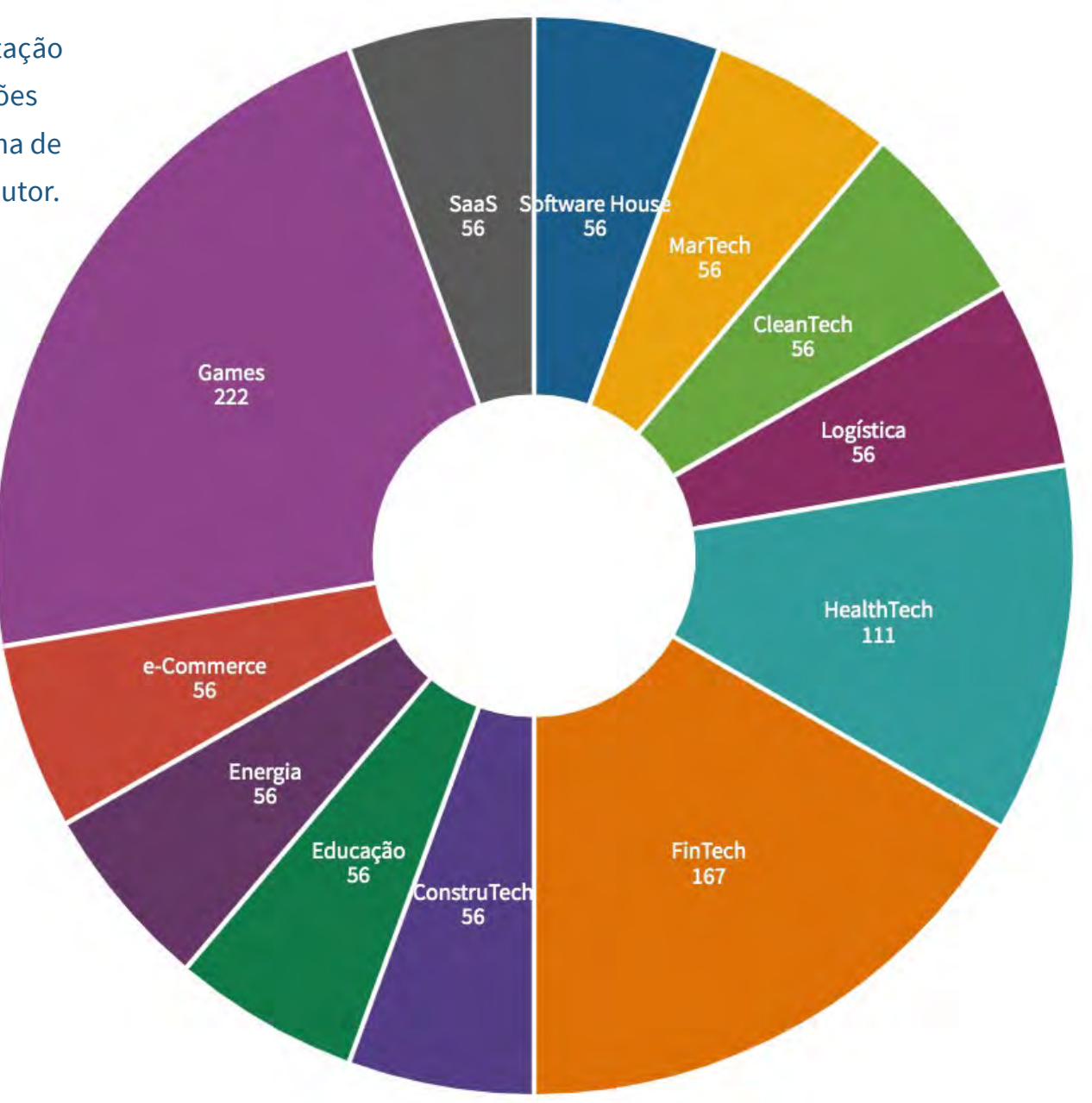

A mudança para uma construção orientada pela narrativa veio como uma evolução deles, também usadas para reforçar a retórica. A mídia impressa hoje prefere usar a infografia narrativa em vez de charts, por serem mais envolventes e sedutoras, e por servirem muito melhor à função desejada desses elementos para os fins editoriais. Segundo o Manual de Redação da Folha de S. Paulo, em sua edição de 1992,

Tudo o que puder ser apresentado na forma de tabelas, mapas, quadros e gráficos não deve ser editado em forma de texto. A tendência do jornalismo é a utilização cada vez maior de artes, principalmente coloridas, que atraem mais o leitor que o texto (FOLHA DE S. PAULO, 1992, p. 122). 
Mas os charts, por mais abstratos e antiquados que sejam, precisam ser lembrados por algo muito importante: a capacidade de lê-los é considerada uma forma de alfabetização básica, ensinada como uma habilidade fundamental nas escolas. São, como já foi dito, uma operação cognitiva per se, que cruza, intersecciona, compara, identifica e diferencia dados, entre vários outros tipos de associações e possíveis relações que podem ser feitas com eles. Usam uma linguagem universal para se expressar: matemática; e embora isso não signifique uma forma superior de comunicação, permite certa portabilidade, interoperabilidade e trocas de informações.

O ponto que tentamos reforçar aqui é que não deveríamos considerar os charts como um formato desatualizado para dar sentido às informações. Eles continuam servindo adequadamente para in[-]formar, sobretudo em alguns contextos e situações específicas.

\section{A ABORDAGEM DA} INFOGRAFIA CENTRADA NA NARRATIVA

Storytelling, ou a inclusão de uma camada narrativa nos artefatos projetados, se tornou uma questão onipresente. Todo produto precisa contar sua história agora, e este requisito também se faz para todo design gráfico ou de interação, que precisarão, igualmente, contar uma para seus usuários. Mesmo uma configuração gráfica sob uma regra estética abstrata implacável tentará contar uma história conceitual ou esquemática, de alguma forma. 


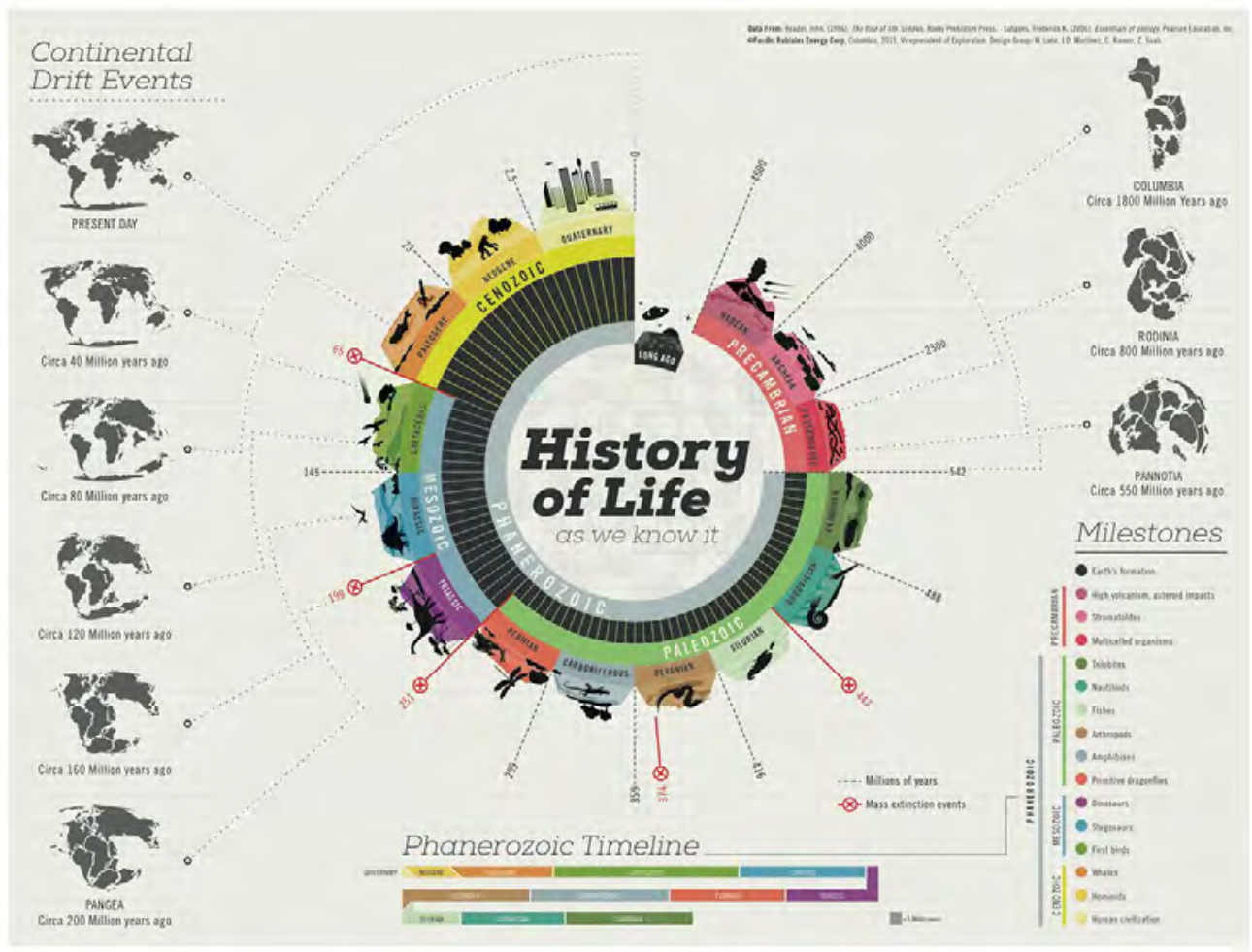

Figura 2: Exemplo de infografia com ênfase narrativa criada pelo designer Juan Martinez. Fonte: portfolio online do autor, <https:// www.behance.net/ gallery/10901127/ History-of-Life>, acesso em 13/6/2021.
Tufte - e não apenas ele - rejeitou a maioria das formas pictóricas e figurativas de elaboração e exibição de informações. Ele as qualificou como "chartjunk" (lixo gráfico):

Esconder-se atrás do chartjunk é desprezo pela informação e pelo público. Os promotores do chartjunk imaginam que os números e os detalhes são enfadonhos, monótonos e tediosos, exigindo ornamentos para animar. A decoração cosmética, que frequentemente distorce os dados, nunca salvará uma falta subjacente de conteúdo. Se os números são enfadonhos, então você está com os números errados. A credibilidade se esvai em nuvens de lixo gráfico; quem confiaria em um gráfico que se parece com um videogame?22 (TUFTE, 1990, p. 34).

02 "Lurking behind chartjunk is contempt both for information and for the audience. Chartjunk promoters imagine that numbers and details are boring, dull, and tedious, requiring ornament to enliven. Cosmetic decoration, which frequently distorts the data, 
Contudo, contra seus melhores desejos, muitas pessoas o farão. E, como bem se observa no artigo de Ricardo Cunha Lima "Metáforas e gráficos pictórico-esquemáticos de Nigel Holmes",

Um dos principais problemas do argumento de Tufte (1984) (1990) sobre os elementos pictóricos metafóricos em gráficos, usados por Holmes e outros designers, é que se trata de uma conclusão que não é embasada em uma análise empírica. Em um estudo recente, Bateman (Bateman et al., 2010), decidiu testar as afirmações de Tufte, comparando os chartjunks pictóricos de Holmes com gráficos estatísticos sem complementação pictórica. A pesquisa de Bateman e de sua equipe consistiu em testes de compreensão e rememoração. $O$ resultado final da pesquisa demonstrou que os participantes identificaram as mensagens de valor numérico nos gráficos de Holmes, significativamente, mais vezes do que nos gráficos simplificados. Além disso, os participantes consideraram os gráficos de Holmes mais atraentes, mais divertidos e descobriram que eram mais fáceis e mais rápidos de lembrar. (LIMA, 2019, p. 336)

As discussões sobre eficácia, relevância e pertinência dentre as duas abordagens (charts e narrativas) continuam vivas até o momento atual, e se estendem também ao dataviz, considerado uma espécie de herdeiro ou aprimoramento dos charts, como defendidos por Tufte. Implicitamente, tentamos argumentar que ambas atendem a necessidades diferentes, e são apenas escolhas estratégias para uma retórica que vai além da construção verbal. Normalmente, esse debate gira em torno da credibilidade dos dados ou do uso de informações (sérias) como forma de entretenimento. Como apontado,

will never salvage an underlying lack of content. If the numbers are boring, then you've got the wrong numbers. Credibility vanishes in clouds of chartjunk; who would trust a chart that looks like a video game?" (tradução do autor) 
enquanto a representação em forma de charts (e suas ramificações) trabalham próximos ao espectro matemático das operações cognitivas, as narrativas trabalham no espectro linguístico, decodificando significados já incluídos na mensagem pelo emissor. Usando o conceito de McLuhan (1964) de mídias frias e quentes, podemos fazer uma aproximação conceituando charts e dataviz como infográficos frios, e os tipos narrativos como infográficos quentes.

\section{A INFOGRAFIA NA MÍDIA ALGORÍTMICA}

Dataviz não começou com computadores. Uma de suas pioneiras foi a enfermeira Florence Nightingale, notavelmente referenciada por seu diagrama de área polar de 1855. Mas a adoção intensa da visualização de dados veio com o aproveitamento do poder computacional para traçar imagens complexas e coloridas de conjuntos de dados também complexos e intrincados; algo que seria considerado muito difícil de ser feito por nossas próprias mãos (e um mouse) com um editor de imagens ou um software de publicação editorial.

Enquadrar esses tipos de imagens geradas por computador como hipermídia ou multimídia não qualifica adequadamente sua ontologia. Hipermídia ou multimídia são termos usados para caracterizar suportes digitais capazes de incorporar uma infinidade de tipos de mídia e conectá-los de forma não linear.

A visualização de dados nos meios digitais é construída a partir de algoritmos; ela se torna código computacional, e é metamórfica em sua essência como tudo nessa mídia.

A desmaterialização é uma operação na qual uma coisa é destituída de seu meio, sendo considerada apenas a sua essência como sendo a coisa, e tomado seu invólucro descartável. O jornal por exemplo, com a internet sofreu a desmaterialização; Ver o jornal hoje significa ler suas notícias em qualquer meio que seja (no próprio jornal, na tela de um computador, no celular, ou impressas em uma folha a parte). $O$ jornal foi reduzido 
a sua essência, e seu meio tornou-se descartável. [...] O meio foi substituído pela noção de interface. Ela é responsável apenas por materializar a coisa de uma maneira conveniente em um determinado momento é portanto perecível, sujeita a sua própria obsolescência, e desmembrada da coisa em si. A interface pode ser modificada conforme a conveniência para que não mate a coisa em seu próprio envelhecimento: é a maneira que produzimos para tornar a essência eterna - descolando-a da casca perecível. O livro envelhece, mas não seu texto. (RANOYA, 2004, p. 31)

Lidar com isso é uma tarefa difícil para designers treinados na criação de formas definitivas para todas as coisas. As visualizações não tem forma, mas regras. Suas regras (código computacional) produzem formas incidentais, e isso é (compreensivelmente) difícil de projetar sem trabalhar o próprio código. Além disso, os algoritmos são alimentados por conjuntos de dados que mudam com o tempo e sobre os quais temos pouco controle. Não há como prever quais dados serão recebidos e colocados na visualização.
Figura 3: Codex

Atlanticus, mapeamento

e visualização dos

escritos e desenhos

de Leonardo Da

Vinci. Fonte: <https://

codex-atlanticus.it/>, acesso em 13/6/2021.

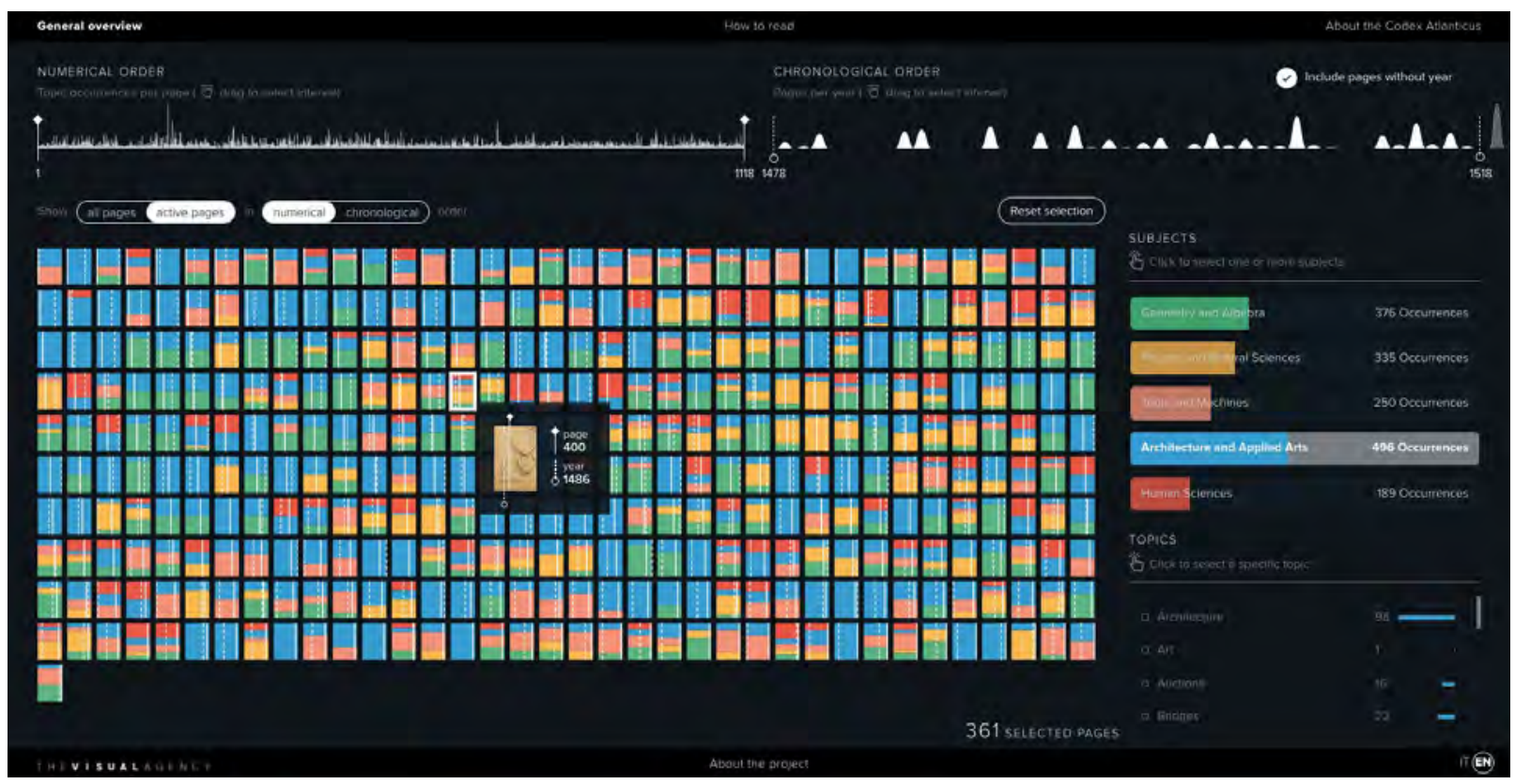


No artigo seminal sobre design de jogos "MDA: a formal approach to game design and game research", Robin Hunicke, Marc LeBlance Robert Zubek explicam o modelo conceitual de como um videogame é gerado, que é necessariamente o mesmo modelo que rege o dataviz e toda visualização gerada por computador. Os game studies têm muitas conexões com dataviz, uma vez que um videogames podem ser entendidos como uma forma frenética de visualização de dados gerada em tempo real. Tufte se preocupava com "quem confiaria em um gráfico que parece um videogame?", mas hoje, cada um deles se comporta como um videogame em seu âmago.

MDA significa "mechanics, dynamics and aesthetics", que podem ser traduzidos como regras, dinâmicas e materialidades. Como explicam seus criadores,

As regras descrevem os componentes específicos do jogo, no nível de representação de dados e algoritmos. A dinâmica descreve o comportamento em tempo de execução da mecânica/regras agindo nas entradas do jogador e nas saídas uns dos outros ao longo do tempo. A 'estética'/materialidade descreve as respostas emocionais desejáveis evocadas no jogador, quando ela interage com o sistema de jogo. ${ }^{03}$ (HUNICKE, LeBLANC \& ZUBEK, 2004, p. 2).

Em termos simples, a mecânica, como as regras definidas por um código; a dinâmica, como a gama de comportamentos e resultados potenciais que essas regras podem produzir; e a estética, como a expressão multimídia decorrente dessa dinâmica. $\mathrm{O}$ que os autores equacionam no modelo, e que é válido para tudo que é diretamente dependente do código computacional, é que a estética/

03 "Mechanics describes the particular components of the game, at the level of data representation and algorithms. Dynamics describes the run-time behavior of the mechanics acting on player inputs and each others' outputs over time. Aesthetics describes the desirable emotional responses evoked in the player, when she interacts with the game system." (tradução do autor) 
materialidade depende da dinâmica e a dinâmica depende das regras/mecânica; os designers criam as regras, enquanto os usuários experimentam as materialidades/estética; os designers não podem moldar a materialidade/estética diretamente, da mesma forma que o usuário não pode compreender as regras/mecânicas imediatamente. Esta é a lógica subjacente para qualquer mídia algorítmica. Podemos usar ferramentas para tentar driblar essas barreiras, sejam softwares que produzem código computacional para o usuário (o que tem sido discutido como programação low-code), ou softwares de edição gráfica. Mas em nenhum destes casos estamos operando a mídia algorítmica em toda a sua capacidade computacional; os resultados produzidos com ferramentas (e não através do código) são extremamente limitados (e limitantes) em termos de agenciamento da máquina computacional: eles conseguem expressar apenas o que a própria ferramenta é capaz de fazer, e não o que a computação ou a esta mídia algorítmica é.

Dataviz não produz imagens estáticas. Pelo contrário, traz um tipo de informação muito dinâmica, como os videogames, pois os dados e as formas de processá-los mudam constantemente. Projetar uma visualização de dados para imitar o comportamento de mídia não dinâmica, para traçar um diagrama final e definitivo, é contraproducente e uma contradição. Isso vai contra sua natureza, mesmo que sua natureza não impeça tal coisa - e isso se tornou um lugar comum - considerando que o suporte multimidiático pode permitir quase qualquer tipo de formato de mídia dentro dela.
É claro que existe o caso do "Big Data", e que para traçarmos uma representação gráfica de um simples chart com a escala de dados deste contexto, por mais final e definitivo que seja, exigirá que a tarefa seja executada por um algoritmo. Esta tarefa poderia ser realizada apenas uma vez, gerando um arquivo de imagem que pode ser reproduzido sem sobrecarregar processadores e computadores. Processamento desnecessário implica desperdício de energia e impactos recalcitrantes na natureza, acumulando-se a cada acesso de cada usuário ao algoritmo, além de aproveitar parte dos recursos e desempenho do próprio computador do usuário que poderiam ser utilizados em outras tarefas, ou garantindo maior conforto e responsividade para ele no que ele está fazendo. Em suma, do ponto de vista da engenharia, é preciso ter economia com recursos computacionais e usar o dataviz para lidar com informações que precisam ser atualizadas constantemente, e não porque adotá-lo seria uma forma engenhosa de se construir um artefato de informação.

As visualizações podem se tornar peças gráficas de grande beleza plástica. E também podem interagir, seja detalhando o que é apresentado ou reorganizando o que dão visualidade de acordo com o interesse do usuário. Apenas com base nessas questões pode-se justificar sua adoção como uma forma de navegar e dar sentido às informações. Então, por que não podemos misturar os dois mundos, unindo a abordagem comunicacional orientada por narrativas, 
e a lógica da mídia algorítmica, com suas regras, dinâmicas e expressões resultantes?

Isso pode ser até possível, mas muito trabalhoso e caro. Os dados presentes em um banco de dados podem ser vastos e imprevisíveis, e o roteirista de uma narrativa precisa conhecer toda a gama de resultados possíveis para criar metáforas e interações adequadas para essa história aberta. Esforços semelhantes já foram tentados através de jogos de computador que trabalham com narrativa interativa, mas que na prática acabam apenas "enfeitando" suas histórias com interações de pequenas consequências sobre a narrativa, como afirma Chris Crawford em seu ensaio sobre "Interactive storytelling": "os resultados, no entanto, não realizaram os grandes sonhos."04 (CRAWFORD, 2003, Kindle Position 6721). As histórias não são moduladas ou alteradas drasticamente pelas interações do usuário. Isso porque criaria uma imensa árvore de ramificações da narrativa, impossível de se resolver em uma solução coerente ou viável. Por fim, as narrativas geralmente são usadas para reforçar uma retórica, e uma forma aberta com resultados imprevisíveis não poderia garantir esta construção. "A maior falácia da 'narrativa interativa' é que ela pretende dar liberdade ao jogador enquanto mantém a coerência narrativa”05 (FRASCA, 2003, pos. 5865).

04 "the results, however, have not realized the grand dreams." (tradução do autor)

05 "The biggest fallacy of 'interactive narrative' is that it
Gonzalo Frasca, um dos pioneiros no campo dos game studies, identifica o fator mais significativo para que a abordagem narrativa e o universo algorítmico não consigam se entremear: é impossível garantir uma narrativa quando há franca liberdade dos atores (que neste caso é a própria informação variável ou a forma como interagimos com ela).

Frente a tantas limitações e dificuldades para trazer à infografia aquilo que a computação poderia lhe prover de melhor, os infográficos digitais acabam recebendo melhorias consideradas cosméticas e ornamentais, como animação e filtros visuais, que, para todos os efeitos, conseguem dar um pouco de vida a estes objetos. Essas são grandes adições, mas muito longe do que o dataviz realmente poderia se tornar, e nesse sentido, estes artefatos se distanciam cada vez mais de sua própria natureza, conforme vão sendo confeccionados através de ferramentas e aproveitando pouco da força computacional inerente. Na direção oposta, está aquilo que a filosofia da imagem discute como regime de simulação (PARENTE, 1999).

\section{SIMULAÇÕES}

"Simular é modelar um sistema (fonte) através de um sistema diferente que mantém (para alguém) alguns dos comportamentos

pretends to give freedom to the player while maintaining narrative coherence" (tradução do autor) 
do sistema original"06 (FRASCA, 2003, Kindle Position 5740), onde a questão-chave aqui é o comportamento, ou mais exatamente, a dinâmica da estrutura do MDA.

Comecemos esta discussão com exemplos práticos. A primeira figura abaixo é uma imagem estática de um vídeo do professor Dan Burns na Los Gatos High School, em 2012, explicando a teoria da relatividade geral, e mais especificamente, como a massa pode dobrar o espaço criando gravidade, um conceito muito sofisticado da astrofísica moderna para alunos do ensino médio. Ele usa um tecido de lycra para simular a tessitura do espaço e o que acontece quando um corpo denso é colocado nele, mostrando todos os pequenos corpos gravitando ao seu redor.
Figura 4: Dan Burns rodando sua simulação física da massa dobrando o espaço. Fonte: <https:// www.youtube.com/ watch?v=MTY1KjeoyLg>, acesso em 13/3/2021.

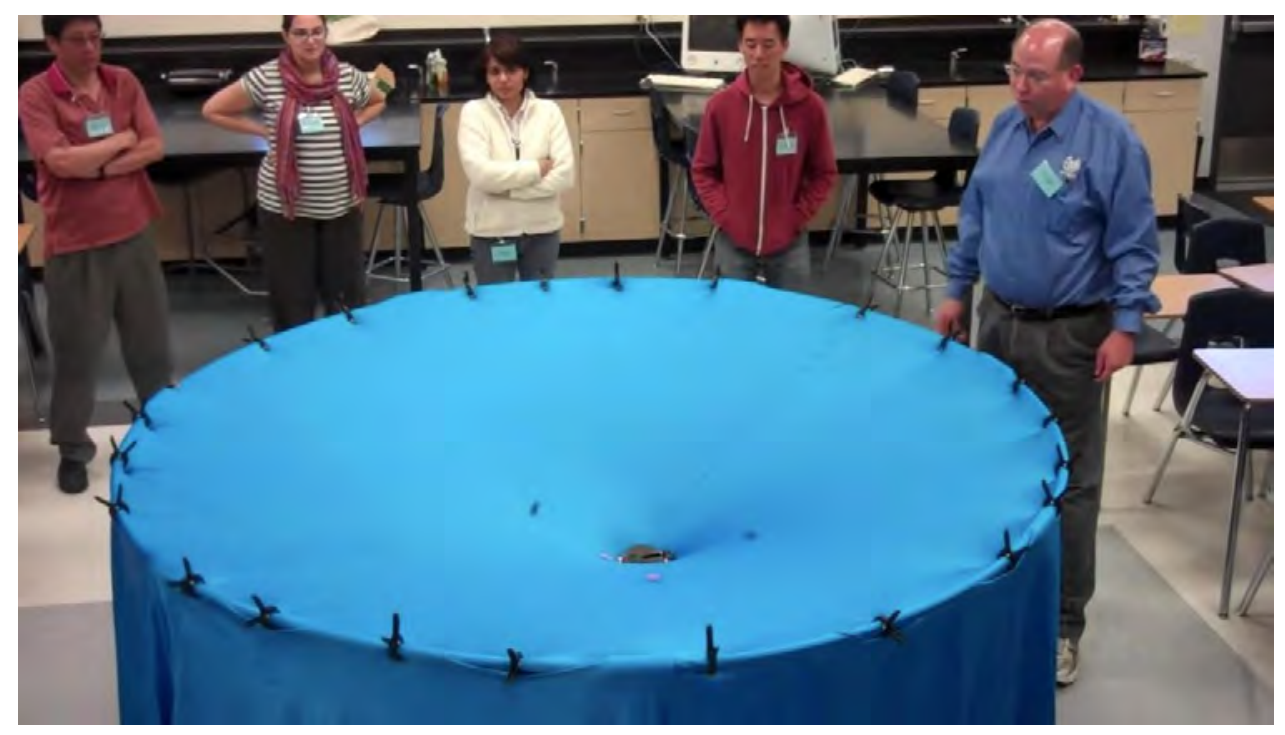

Seu experimento tangível transforma uma formulação complexa bem conhecida em algo simples de entender. Enquanto seus alunos observam a gravitação acontecendo e entendem as regras dessa força da natureza, eles podem até prever o que acontecerá em alguns momentos. A simulação é uma forma de conhecimento. Ou, talvez, a simulação forme conhecimento. É uma exploração de como as coisas poderiam ser, mesmo que não sejam. Não como uma projeção baseada em suposições ou expectativas, mas na experimentação de

06 "To simulate is to model a (source) system through a different system which maintains (for somebody) some of the behaviours of the original system" (tradução do autor) 
uma virtualidade. Esse "acontecer sem ser" é precisamente o que Pierre Lévy chama de virtualidade:

Já o virtual não se opõe ao real, mas sim ao atual. Contrariamente ao possível, estático e já constituído, o virtual é como o complexo problemático, o nó de tendências ou de forças que acompanha uma situação, um acontecimento, um objeto ou uma entidade qualquer, e que chama um processo de resolução: a atualização. Esse complexo problemático pertence à entidade considerada e constitui inclusive uma de suas dimensões maiores. $O$ problema da semente, por exemplo, é fazer brotar uma árvore. (LÉVY, 1996, p. 7)

O segundo exemplo, apresentado pelas duas figuras abaixo, é de um dos muitos artigos em uma publicação científica chamada Distill, de-

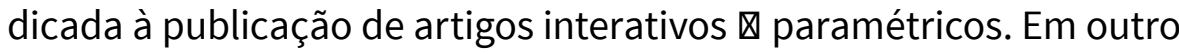
artigo, também disponível lá e discutindo a publicação $\bigotimes$ plataforma em si, Fred Hohman, Matthew Conlen, Jeffrey Heer e Duen Horng (Polo) Chau, dizem que :

Hoje há um entusiasmo crescente em torno do uso de artigos interativos para comunicação, uma vez que eles oferecem recursos exclusivos para ajudar as pessoas a aprender e se envolver com ideias complexas que faltam na mídia tradicional. [...] Este estilo de comunicação - e as plataformas que o suportam - ainda estão em sua infância. Ao escolher onde publicar este trabalho, queríamos que o meio refletisse a mensagem. Periódicos como o Distill não estão apenas expandindo os limites da pesquisa em aprendizado de máquina, mas também oferecem um espaço para criar novas interfaces para divulgação. Este trabalho reúne a teoria e a prática de autoria e publicação de artigos interativos. ${ }^{07}$ (HOHMAN, 2020, online).

07 "Today there is a growing excitement around the use of interactive articles for 


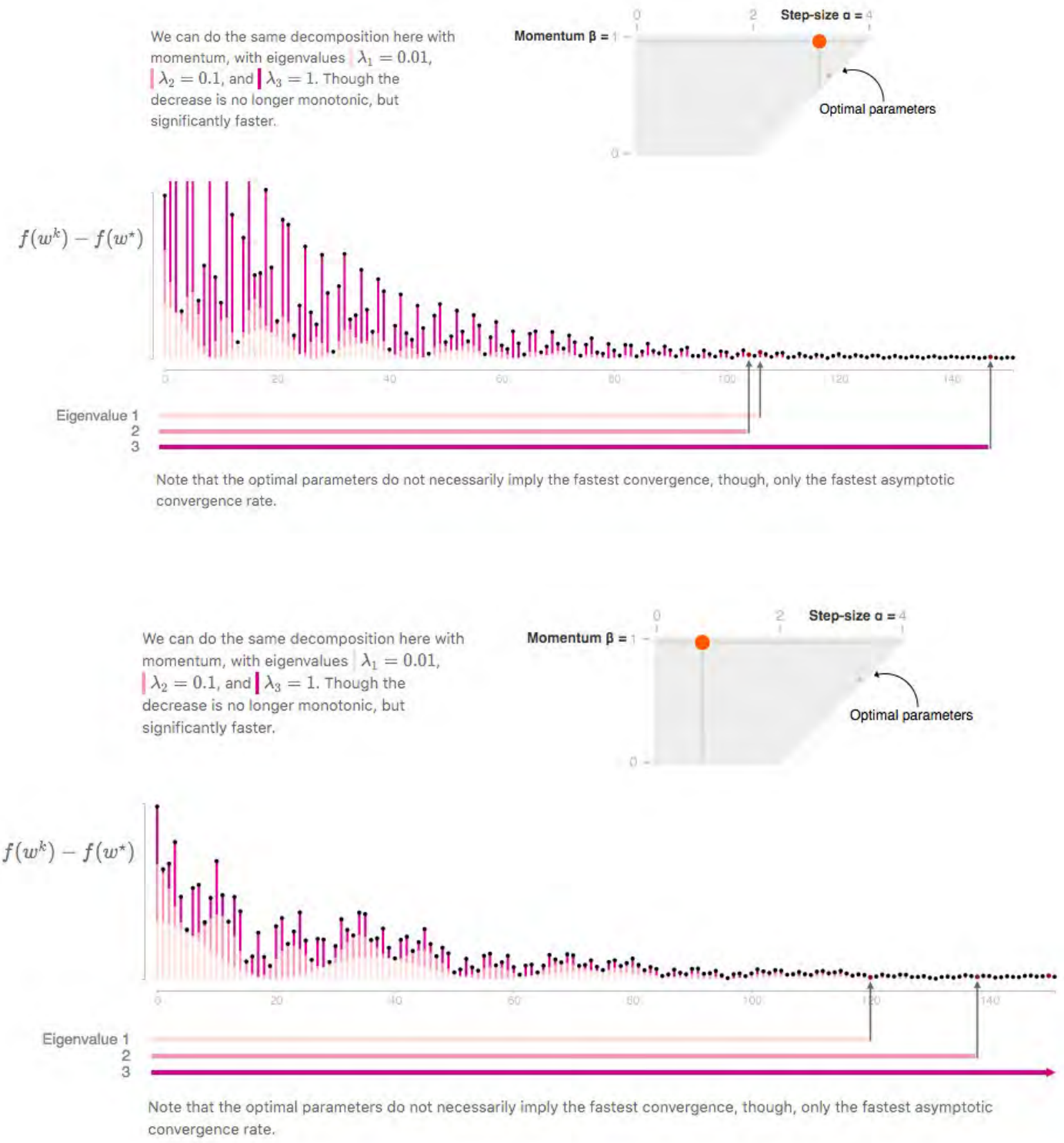

communication since they offer unique capabilities to help people learn and engage with complex ideas that traditional media lacks. [...] This style of communication - and the platforms which support it - are still in their infancy. When choosing where to publish this work, we wanted the medium to reflect the message. Journals like Distill are not only pushing the boundaries of machine learning research but also offer a space to put forth new interfaces for dissemination. This work ties together the theory and practice of authoring and publishing interactive articles." (tradução do autor)

Figuras 5 e 6. Brincando com diagramas interativos no artigo "Why momentum really works" de Gabriel Goh. Fonte: <https://distill. pub/2017/momentum/>, , acesso em 13/3/2021. fronteiras do design 2. [in]formar novos sentidos 
Ambas as figuras mostram as mudanças em um diagrama gráfico durante a interação com ele. $\mathrm{O}$ artigo de Gabriel Goh, chamado "Why momentum really works" é sobre física e está repleto de fórmulas matemáticas em todo o texto. A excepcionalidade desta publicação é que você pode ver e experimentar as variáveis-chave dentro das fórmulas e acompanhar os resultados de suas alterações. Resumindo, é possível simular diferentes casos para entender seus efeitos. Por meio da simulação, a in[-]formação torna-se quântica: a mesma informação pode assumir posições e resultados diferentes, dependendo de como é inferida; e o dar sentido às coisas é feito naturalmente de inúmeros pontos de vista diferentes, que podem ser modificados para fornecer uma melhor compreensão de seu significado ${ }^{08}$.

Segundo Frasca, em um de seus textos mais referenciados, "Simulation versus narrative: introduction to ludology":

Ao contrário da mídia tradicional, os videogames não se baseiam apenas na representação, mas em uma estrutura semiótica alternativa conhecida como simulação. Mesmo que as simulações e narrativas compartilhem alguns elementos comuns (personagens, cenários e eventos), sua mecânica é essencialmente diferente. Mais importante, eles também oferecem possibilidades retóricas distintas. [...] são representacionais, não simulacionais. Eles se destacam em produzir tanto descrições de traços quanto sequências de eventos (narrativa). Uma fotografia de um avião nos dará informações sobre sua forma e cor, mas não voará ou cairá quando manipulado. Um simulador de vôo ou um simples avião de brinquedo não são apenas sinais,

08 Novamente, não está no escopo deste artigo discutir a credibilidade e fidelidade das informações, mas é muito mais difícil - contudo ainda possível - distorcer as informações por meio de uma simulação. Seria necessário tratar cada condição indesejada caso a caso e criar regras que contemplassem cada uma. Este é um processo excessivamente complicado e caro. Ainda assim, é possível. 
mas máquinas que geram sinais de acordo com regras que modelam alguns dos comportamentos de um avião real. ${ }^{09}$ (FRASCA, 2003, Kindle Position 5701).

Em seu trabalho, o autor argumenta que, historicamente, os jogos (como mídia) têm sido tratados (cientificamente) como uma continuidade das questões narrativas, e "a Ludologia não despreza essa dimensão dos videogames, mas afirma que eles não são mantidos juntos por uma estrutura narrativa"10 (FRASCA, 2003, Kindle Position 5714). Novamente, suas declarações sobre jogos têm muitas semelhanças com os problemas de dataviz. Ele também faz afirmações ousadas dizendo que a simulação está além dos limites da semiótica, e Frasca não é o único. Paola Antonelli, curadora de artefatos de design do Museu de Arte Moderna (MoMA) de Nova York e responsável pela exposição "Talk to me: design and the communication between people and objects", afirma algo semelhante:

Em contraste com o triunfo da semiótica do século XX, que desprezava a comunicação como nada além de uma transmissão mecânica de significado codificado, o século XXI começou como um século de pancomunicação - tudo e todos transmitindo conteúdo e significado em todas as combinações possíveis, de um para um,

09 "unlike traditional media, video games are not just based on representation but on an alternative smiotical structure known as simulation. Even if simulations and narratives do share some common elements (characters, settings, and events) their mechanics are essentially different. More important, they also offer distinct rhetorical possibilities. [...] are representational, not simulational. They excel at producing both descriptions of traits and sequences of events (narrative). A photograph of a plane will tell us information about its shape and color, but it will not fly or crash when manipulated. A flight simulator or a simple toy plane are not only signs, but machines that generate signs according to rules that model some of the behaviors of a real plane." (tradução do autor)

10 "Ludology does not disdain this dimension of video games but claims that they are not held together by a narrative structure" (tradução do autor) 
para tudo, e para todos. Agora esperamos que os objetos se comuniquem, uma mudança cultural que fica evidente quando vemos crianças procurando botões ou sensores em um novo objeto, mesmo quando o objeto não tem bateria ou tomada. 'Talk to me: design and the communication between people and objects' (Fale comigo: o design e a comunicação entre pessoas e objetos) prospera neste importante desenvolvimento do final do século XX na cultura do design, que pode ser descrito como uma mudança da centralidade da função para a do significado, e no foco do primeiro século na necessidade de comunicar para existir. Nessa nova perspectiva, todos os objetos ocupam uma posição única na cultura material e todos eles contêm informações além de seu uso ou aparência imediata. Não é suficiente para os designers hoje equilibrar forma e função, e também não é suficiente simplesmente atribuir significado. [...] como Khoi Vinh aponta [...] As coisas podem se comunicar com as pessoas, mas os designers escrevem o roteiro inicial que nos permite desenvolver e improvisar o diálogo." (ANTONELLI, 2007, p. 7)

11 "In contrast to the twentieth-century triumph of semiotics, which looked down on communication as nothing but a mechanical transmission of coded meaning, the twentyfirst century has begun as one of pancommuniciation - everything and everybody conveying content and meaning in all possible combinations, from one-on-one to everything-on- everybody. We now expect objects to communicate, a cultural shift made evident when we see children searching for buttons or sensors on a new object, even when the object has no batteries or plug. Talk to Me: Design and the Communication between People and Objects thrives on this important late-twentieth-century development in the culture of design, which can be described as a shift from the centrality of function to that of meaning, and on the twenty-first-century focus on the need to communicate in order to exist. From this new perspective, all objects occupy a unique position in material culture, and all of them contain information beyond their immediate use or appearance. It is not enough for designers today to balance form and function, and it is also not enough simply to ascribe meaning. [...] as Khoi Vinh points out [...] Things may communicate with people, but designers write the initial script that lets us develop and improvise the dialogue." 
A refutação de suas proposições afirma que uma sequência de imagens (signos), seja em um filme, jogo ou em uma simulação, terá exatamente a mesma aparência. Mas uma simulação (ou jogo) não pode ser entendida exclusivamente por meio de um de seus resultados infinitos (virtuais). E a semiótica é uma ciência cognitiva que considera apenas o fenômeno percebido (as "atualizações" nos termos de Lévy), portanto, intrinsecamente limitado em um contexto virtual.

Costuma ser difícil aceitar que haja uma alternativa à representação e à narrativa: a simulação. [...] No final da década de 1990, Espen Aarseth revolucionou os estudos de texto eletrônico com a seguinte observação: os textos eletrônicos podem ser mais bem compreendidos se analisados como sistemas cibernéticos. Ele criou uma tipologia de textos e mostrou que o hipertexto é apenas uma das dimensões possíveis desses textos sistêmicos, que ele chamou de 'cibertextos'. A teoria literária tradicional e a semiótica simplesmente não podiam lidar com esses textos, jogos de aventura e ambientes multiusuário baseados em texto porque essas obras não são feitas apenas de sequências de signos, mas, ao contrário, se comportam como máquinas ou geradores de signos. O reino da representação foi contestado academicamente, abrindo caminho para simulação e estudos de jogos. ${ }^{12}$ (FRASCA, 2003, Kindle pos. 5725).

(tradução do autor)

12 "is usually difficult to accept that there is an alternative to representation and narrative: simulation. [...] In the late 1990s, Espen Aarseth revolutionized electronic text studies with the following observation: electronic texts can be better understood if they are analyzed as cybernetic systems. He created a typology of texts and showed that hypertext is just one possible dimension of these systemic texts, which he called 'cybertexts.' Traditional literary theory and semiotics simply could not deal with these texts, adventure games, and textual-based multiuser environments because these works are not just made of sequences of signs but, rather, behave like machines or sign-generators. The reign of 
Com base nessa linha de raciocínio, também é possível entender a simulação como uma forma diferente das abordagens dos charts, e das abordagens narrativas, e ainda assim, capaz de conciliar uma parte razoável das características de cada uma delas, embora não possa ser considerada uma história, ou reduzida à operação simbólico-cognitiva de cruzamento, interseção, etc.

\section{CONSIDERAÇÕES FINAIS}

Isso é o que dataviz poderia ser, mas ainda não é. Oferecendo contra-argumentos, pode-se dizer que não se trata de uma discussão sobre visualização de dados, mas sobre a mídia algorítmica, ou seja, que a visualização precede a mídia algorítmica, e é independente dela, existindo, por exemplo, na mídia impressa; que não estamos discutindo aqui sobre dataviz, mas sobre qual é a ontologia dessa nova mídia. Também poder-se-ia dizer que a visualização de dados não pode ser confundida com a interação com dados, ou com a simulação através deles, sendo cada um desses tópicos uma frente inteiramente nova de discussão.

Sim, talvez tudo isso seja também verdadeiro. Uma disruptura se estabeleceu conforme a tecnologia da informação e a representação de dados se aproximaram, e se juntaram como se fossem uma coisa só. E não devemos esquecer que a própria representação gráfica é um tipo - muito antigo - de tecnologia da informação que nasce do encontro das técnicas do desenho com as novas ideias de como apresentar a informação.

Então, o que dataviz poderia ser, no sentido da filosofia do virtual, não pode garantir o que se tornará. Muitas coisas podem ser diferentes do que realmente são; a semente pode não se tornar uma árvore, ou pode até se tornar um pequeno arbusto. Isso não depende de sua natureza, mas dos acontecimentos que a cercam e de seu desenvolvimento diante deles. Nossa discussão aqui é centrada em

representation was academically contested, opening the path for simulation and game studies." (tradução do autor) 
torno do conceito filosófico de potência, e nesse momento, o que se observa é que esta árvore em especial não está encontrando as condições para se diferenciar das demais, ou para se afirmar como uma espécie singular no seu ecossistema.

Dataviz tem a potência de se tornar uma forma de simulação, e a potência de se tornar uma forma muito rica de dar sentido às coisas por meio dela. Como Flusser apontou, in[-]forma[-]ação é a "forma" dada a algum "o quê", e o "o quê" é realmente aquilo que está em jogo. Se há uma aposta, também há um jogo a ser disputado, e a informação é o campo para essa disputa sobre o significado das coisas. Em condições virtuais, a informação não tem forma, ou para ser mais preciso, tem muitas formas e não uma única fixa e definitiva.

A simulação é uma máquina automatizada que fornece sinais incessantes. E essa sobrecarga simbólica de sua virtualidade transforma completamente nossos processos de produção de sentido. Talvez não estejamos prontos para esse tipo de coisa, mas algo é certo: é difícil configurar artefatos para que permaneçam indefinidos (ou para que sejam metafóricos), e é ainda pior quando os dados (seu "o quê", seu conteúdo) são indeterminados por natureza. Há indeterminações em demasia para serem conciliadas com as práticas com as quais estamos habituados. 


\section{BIBLIOGRAFIA}

ANTONELLI, Paola. Talk to me: design and the communication between people and objects. New York: MoMA, 2011.

CRAWFORD, Chris. "Interactive storytelling" in The video game theory reader. New York: Routledge, 2003. E-book Kindle.

FLUSSER, Vilém. 0 mundo codificado - por uma filosofia do design e da comunicação. São Paulo: Cosac Naify, 2007.

FRASCA, Gonzalo. "Simulation versus narrative: introduction to ludology" in The video game theory reader. New York: Routledge, 2003. E-book Kindle.

FOLHA DE S. PAULO. Novo manual da redação. São Paulo: Publifolha, 1992.

$\mathrm{GOH}$, Gabriel. Why momentum really works, Distill, 2017. https://doi.org/10.23915/distill.00006 . Disponível em https://distill. pub/2017/momentum/, acesso em 23 de maio de 2021.

HOHMAN F.; CONLEN, M.; HEER, J. \& CHAU, D.. Communicating with Interactive Articles, Distill, 2020. Disponível em https://distill. pub/2020/communicating-with-interactive-articles/, acesso em 23 de maio de 2021.

HUNICKE, R.; LeBLANC, M. \& ZUBEK, R. MDA: a formal approach to game design and game research, Proceedings of the Challenges in Games AI Workshop, Nineteenth National Conference of Artificial Intelligence, 2004. 
LÉVY, Pierre. 0 que é o virtual. São Paulo:

Ed. 34, 1996.

LIMA, Ricardo Cunha. Metáforas e gráficos pictórico-esquemáticos de Nigel Holmes | Nigel Holme's metaphors and pictorial-schematic charts. InfoDesign - Revista Brasileira De Design Da Informação, 16(3), 330-348. https://doi.org/10.51358/ id.v16i3.772, 2019.

MCLUHAN, Marshal. Os meios de comunicação com extensões do homem. São Paulo: Cultrix, 1964.

PARENTE, Andre. 0 virtual e o hipertextual. Rio de Janeiro: Pazulim, 1999.

RANOYA, Guilherme. Tecnologias da desmaterialização. Novos Olhares, (13), 22-35, 2004. https://doi.org/10.11606/issn.2238-7714. no.2004.51394

WITTGENSTEIN, Ludwig. Tratado lógico-filosófico. Lisboa: Calouste Gulbenkian, 1987.

WOLF, Mark J.P. \& PERRON, Bernard. The video game theory reader. New York: Routledge, 2003.

TUFTE, Edward. The visual display of quantitative information. Cheshire: Graphic Press LLC, 2001.

TUFTE, Edward. Envision information. Cheshire: Graphic Press LLC, 1990. 
259 